\title{
Leukocyte ROS Measured with Flow Cytometry before and after a Strength Training Session
}

\author{
Hilde G Nielsen ${ }^{1,2 *}$ and Torstein Lyberg ${ }^{2}$ \\ ${ }^{1}$ The Research Council of Norway, Lysaker, Norway \\ ${ }^{2}$ Department of Medical Biochemistry, Oslo University Hospital, Ullevål, Norway
}

Submission: July 23, 2018; Published: August 02, 2018

*Corresponding author: Hilde G Nielsen, The Research Council of Norway, 1327 Lysaker, Norway, Tel: +47 409222 60;

Email: hildegnielsen@outlook.com

\begin{abstract}
According to the literature there is a link between the intensity of exercise and the occurrence of infections and disease. Strength training is physical exercise with the use of resistance to induce muscular contraction. Reactive oxygen species (ROS) are unstable molecules that contains oxygen and are formed in the body. Antioxidants are important to prevent chemical damage to the cell components by oxidation. Oxidative stress is defined as a disturbance in the balance between the production of ROS and antioxidant defenses. The apprehension of increased rates of ROS production during exercise is part of a rationale why many athletes ingest antioxidant supplements beyond recommended dose. The aim of this present study was to investigate changes in leukocyte levels of ROS measured with flow cytometry, total antioxidant status (TAS) and catalase before and after a strength training session. Ten men and ten women were recruited to this study. All participants went through a strength training session consisting of nine exercises executed at $80 \%$ of $1 \mathrm{RM}, 6-8$ repetitions $/ 3$ sets. The labelled samples were analysed within $24 \mathrm{~h}$ in a flow cytometer. Examining the post-training blood samples, they were all non-significantly changed compared to the pre-training samples. The TAS and the levels of plasma catalase did not change as a result of the training session. The strength training session were not enough to influence the leukocyte production of ROS and the total antioxidant system in trained students. Based on these findings, there is no rationale to advice athletes to ingest antioxidant supplementation in addition to their regular diet.
\end{abstract}

Keywords: Leukocytes; Reactive oxygen species; Total antioxidant status; Strength training

Abbreviations: ROS: Reactive Oxygen Species; TAS: Total Antioxidant Status; 1RM: One Repetition Maximum; PMA: Phorbol Myristate Acetate; WBC: White Blood Cell; PBS: Phosphate- Buffered Saline; MFI: Mean Fluorescence Intensity; TAS: Total Antioxidant Status

\section{Introduction}

Physical exercise can enhance or maintains physical fitness and overall health and wellness [1-4]. According to the literature, there is a link between the intensity of exercise and the occurrence of infections and disease [1,5-8]. Regular physical exercise and exercise at moderate levels are important factors for disease prevention $[1,9]$.

Strength training is physical exercise with the use of resistance to induce muscular contraction. The effects of the training are increased muscle strength, anaerobic endurance and increased size of skeletal muscles. Strength training is dependent of the amount of work required to achieve the activity of the training (intensity), number of muscles worked, exercise, sets and reps (volume) and how many training sessions performed per week (frequency) [3,4].

Reactive oxygen species (ROS) are formed in the body. ROS include oxygen ions, free radicals and peroxides, both inorganic and organic. Both granulocytes and monocytes produce ROS, e.g superoxide anion $\left(\mathrm{O}_{2}^{-}\right)$, hydrogen peroxide $\left(\mathrm{H}_{2} \mathrm{O}_{2}\right)$, peroxinitrite $\left(\mathrm{ONOO}^{-}\right)$and hydroxyl radical $(\bullet \mathrm{OH})$. Superoxide anions are unstable free radicals that kills bacteria directly and is generated both in intra- and in extracellular compartments. Nitric oxide and $\mathrm{O}_{2}^{-}$react with each other and form rapidly ONOO- Peroxinitrite is a strong radical, which can damage DNA, proteins and other cellular elements. Many sources of heat, stress, irradiation, inflammation and any increase in metabolism, including exercise lead to increased production of ROS [10-12]. The uncertainty of increased rates of ROS production during exercise is part of a rationale why many athletes ingest antioxidant supplements beyond suggested doses $[9,11,13,14]$.

We have used the highly sensitive flow cytometric method for measuring ROS generation in leukocytes. Using fluorescence probes and flow cytometry we have earlier shown that the phorbol ester phorbol myristate acetate (PMA) may induce an 
increase in the levels of ROS in granulocytes by at least a factor of 150 [15]. In endurance running, e.g. marathon race, we could show that the capacity of leukocytes (both granulocytes and monocytes) to respond with ROS after in vitro PMA stimulus was heavily suppressed [16]. A tendency in this direction also applied for a half-marathon race [16] whereas a short- term maximal physical exercise (test of maximal oxygen consumption $\left.\left(\mathrm{VO}_{2 \max }\right)\right)$ did not induce such a suppression of ROS generation, but instead a significant increase in intracellular granulocytes ROS following PMA stimulation [17].

Antioxidants are important to prevent chemical damage to the cell components by oxidation. An antioxidant is a chemical compound and is thought to defend the body cells from the destructive effects of oxidation (ROS). Catalase is an enzyme found in nearly all living organisms exposed to oxygen (such as bacteria, plants, and animals). Catalase decompose hydrogen peroxide to water and oxygen. Catalase is a very important enzyme in protecting the cell from oxidative damage by reactive oxygen species (ROS). Antioxidants and training prevent against ROS production in exhaustive exercise [5,9].

To compare with our different historical groups on running exercises, the aim of the present study was to investigate in athletes the changes in leukocyte levels of ROS measured with flow cytometry, total plasma antioxidant capacity and catalase before and after a strength training session.

\section{Materials and Methods}

\section{Subjects}

Ten men and ten women were recruited to this study. The average age of the men were 25 years old (range 22-28), and the average age of the women were 26 years old (range 23-30). The participants were all students and were recruited in an exercise room at the Oslo university campus. The men trained in average 3,6hours/week, while the women trained in average 3,9hours/ week. The average time of each training sessions were for the men 1,8hours/time (range 1,5 - 2,5hours/time) and 1,6hours/ time (range 1,0 - 2,5hours/time) for the women. The main exercise was in addition to strength training, endurance training as running, bicycling, spinning and football. All participants were asked to avoid training the day before the test. None of the participants ingested vitamins regularly, but two participants eat cod liver oil daily. The subjects were informed about the study and gave their written informed consent for participation. The study was approved by the Regional Committee for Medical and Health Research Ethics (REC) before the start of the project.

\section{Study Design/Blood Samples}

Venous blood samples were taken before (before the warm up) and immediately after the training session (within 10 minutes). Venous blood was sampled into anticoagulated (EDTA or heparin) vacuum tubes (Becton Dickinson, Plymouth, UK). The blood samples were kept on ice and transported within
30 minutes to the appropriate laboratory at Oslo university hospital, Ullevål.

\section{Test Protocols}

After 20 minutes warm up running at a treadmill or biking, all participants went through a strength training session consisting of nine exercises; squats, bench press, shoulder press, biceps, curl, leg curve, leg extension, back and belly. Every exercise was done at about $80 \%$ of One Repetition Maximum (1RM) with six to eight repetitions and three sets.

\section{Hematological Analyses}

White blood cell (WBC) and platelet counts, hemoglobin and hematocrit were determined in EDTA blood using the Technicon $\mathrm{H}_{2} \bullet^{\text {TM }}$ System (Bayer, Tarrytown, NY, USA) at the Department of Medical Biochemistry, Oslo University Hospital, Ullevål.

\section{Preparation of Blood Leukocytes for Flow Cytom- etry}

\section{Basal (ex vivo) ROS Levels}

Aliquots of $50 \mu \mathrm{l}$ EDTA whole blood were incubated for $15 \mathrm{~min}$ at $37^{\circ} \mathrm{C}$ in a $5 \% \mathrm{CO} 2 /$ humidified air atmosphere in $5 \mathrm{ml}$ polystyrene round-bottom tubes (2052 Falcon, Oxnard, CA, USA) with dihydroethidium (DHE) (Sigma, St. Louis, MO, USA) (final concentration $5 \mu \mathrm{mol} / \mathrm{l}$ ), recognizing mainly the oxygen species superoxide anion $\left(\mathrm{O}_{2}^{-}\right)(18)$ or with dihydrorhodamine 123 (DHR) (Molecular Probes, Leiden, Netherlands) (final concentration $5 \mu \mathrm{mol} / \mathrm{l})$, recognizing the oxygen species peroxynitrite (ONOO-), hypochlorous acid and hydrogen peroxide (9) or with phosphate- buffered saline (PBS) (Sigma) (10 mmol/l phosphate buffer, $2.7 \mathrm{mmol} / \mathrm{l} \mathrm{KCl,} 137 \mathrm{mmol} / \mathrm{l} \mathrm{NaCl}, \mathrm{pH} 7.4$ ) as autofluorescence control. After ended incubation, $1.5 \mathrm{ml}$ red cell lysing solution containing $156 \mathrm{mmol} / \mathrm{l} \mathrm{NH} 4 \mathrm{Cl}, 10 \mathrm{mmol} / \mathrm{l}$ NaHCO3 and 0,12mmol/l EDTA was added to the tubes. The tubes were incubated in the dark at room temperature for $15 \mathrm{~min}$ and centrifuged at $300 \mathrm{~g}$ for $5 \mathrm{~min}$ at $4^{\circ} \mathrm{C}$. The leukocytes were washed once in $2 \mathrm{ml}$ cold PBS and finally resuspended in $0.5 \mathrm{ml} 1 \%(\mathrm{w} / \mathrm{v}$ ) paraformaldehyde in PBS, $\mathrm{pH}$ 7.4. The samples were stored in the dark at $4^{\circ} \mathrm{C}$ until flow cytometry could be performed (within 24h).

\section{PMA-Stimulated (in vitro) ROS Production}

Aliquots of $1.5 \mathrm{ml}$ EDTA whole blood were incubated in polystyrene tubes with ventilation caps with $15 \mu$ l phorbol myristate acetate (PMA) (final concentration 100ng/ml) (Sigma) or PBS (control) at $37^{\circ} \mathrm{C}$ (DHE) and $30^{\circ} \mathrm{C}$ (DHR) for $60 \mathrm{~min}$ in a $\mathrm{CO}_{2}$ incubator. Aliquots of $50 \mu \mathrm{l}$ blood were then labeled with DHE and DHR as described for basal ROS levels.

\section{Flow Cytometry Analysis}

The labeled samples were analysed within $24 \mathrm{~h}$ in a FACSort $^{\mathrm{TM}}$ flow cytometer (Becton Dickinson, San Jose, CA, USA). The flow cytometer was equipped with an argon laser and 
CellQuest $^{\mathrm{TM}}$ software (Becton Dickinson). Ten thousand events were collected from each sample. The leukocyte subpopulations monocytes and granulocytes, were identified by their light scatter characteristics, enclosed in electronic gates and separately analysed for fluorescence intensity from the separate (DHE/DHR) fluorescent probes. The results are expressed as mean fluorescence intensity (MFI). The intra assay coefficient of variation (CV) was $<5 \%$ in unstimulated and $<10 \%$ in PMAstimulated samples.

\section{Total Antioxidant Status and Catalase}

Blood samples were collected in heparin tubes and centrifuged at $2300 \mathrm{~g}, 4^{\circ} \mathrm{C}$ for $12 \mathrm{~min}$ and plasma stored at $-70^{\circ} \mathrm{C}$ until analysis. The Randox Total Antioxidant Status (TAS) kit (Crumlin, UK) and The Catalase Assay kit (Cayman Chemical, UK) were used according to the manufacturer's instructions.

\section{Statistical Analyses}

Results are given as means and standard errors of the mean (mean, SEM). Difference within groups was analyzed using twotailed paired sample t-test (from pre to post). Independent sample t-test was used in order to compare men and women. Since we did not observe any statistically significant differences between the women and men, we have chosen to present the results as one group (except the hematology values which are presented separately for each group).

\section{Results}

\section{Subjects}

All the twenty subjects completed the training session. One subject did train before the test day, even that they were informed of no training before the test. None of the participants ingested any nutrition supplement the day before strength training session.

\section{Haematology}

The total number of leukocytes was within normal range (3.0-11.0 $\left.\times 10^{9} . \mathrm{L}^{-1}\right)$ before the strength training session. After the session the numbers had increased but not significantly. Both the haematocrit, the haemoglobin and thrombocytes remained at the same level after the training session (Table 1).

Basal and stimulated leukocytes ROS levels before and after the training session Table 2 summarise the separate figures (MFI \pm SEM) for PBS (control) and PMA- stimulated leukocytes and the stimulating index (SI = PMA-stimulated/PBS) for the separate fluorechromes (DHR/DHE) in granulocytes and monocytes, comparing the pre- versus post-training sessions. Using the DHR probe, the stimulation index in granulocytes of pre-training blood samples was $199 \pm 16$ (monocytes $2.1 \pm 0.2$ ) whereas the corresponding figures using the DHE probe was $62 \pm 5$ (monocytes $12.2 \pm 1.0$ ).

Table 1: Haematologic parameters of the subjects before and after the training session. Data are expressed as mean $\pm \mathrm{SEM}$. $\mathrm{N}=20$.

\begin{tabular}{|l|c|l|l|l|}
\hline & \multicolumn{2}{|c|}{ Men (N=10) } & \multicolumn{2}{c|}{ Women (N=10) } \\
\hline & Before & After & Before & After \\
\hline Leukocytes (total) $\left(10^{9} \cdot \mathrm{L}^{-1}\right)$ & $6.7(0.6)$ & $8.6(0.8)^{\mathrm{NS}}$ & $6.2(0.3)$ & $6.6(0.0)^{\mathrm{NS}}$ \\
\hline Neutrophils $\left(10^{9} \cdot \mathrm{L}^{-1}\right)$ & $3.4(0.4)$ & $4.2(0.5)^{\mathrm{NS}}$ & $2.8(0.2)$ & $3.2(0.2)^{\mathrm{NS}}$ \\
\hline Monocytes $\left(10^{9} \mathrm{~L}^{-1}\right)$ & $0.6(0.01)$ & $0.8(0.1)^{\mathrm{NS}}$ & $0.5(0.0)$ & $0.5(0.0)^{\mathrm{NS}}$ \\
\hline Lymphocytes $\left(10^{9} \mathrm{~L}^{-1}\right)$ & $2.6(0.1)$ & $3.4(0.3)^{+}$ & $2.7(0.2)$ & $2.6(0.3)^{\mathrm{NS}}$ \\
\hline Eosinophils $\left(10^{9} \mathrm{~L}^{-1}\right)$ & $0.1(0.0)$ & $0.2(0.0)^{\mathrm{NS}}$ & $0.2(0.1)$ & $0.2(0.1)^{\mathrm{NS}}$ \\
\hline Haemoglobin $\left(\mathrm{g} \mathrm{dL}^{-1}\right)$ & $15.0(0.2)$ & $15.6(0.2)^{\mathrm{NS}}$ & $13.6(0.3)$ & $13.7(0.3)^{\mathrm{NS}}$ \\
\hline Haematocrit $(\%)$ & $0.41(0.0)$ & $0.45(0.0)^{\mathrm{NS}}$ & $0.41(0.0)$ & $0.41(0.0)^{\mathrm{NS}}$ \\
\hline Thrombocytes $\left(10^{9} \cdot \mathrm{L}^{-1}\right)$ & $241(19.8)$ & $286(24.1)^{\mathrm{NS}}$ & $274(10.8)$ & $283(19.7)^{\mathrm{NS}}$ \\
\hline
\end{tabular}

NS $=$ non significance, ${ }^{*} \mathrm{p}=0.05$

Table 2: Basal and PMA-stimulated leukocyte ROS levels before and after the training session. Data are expressed as mean mean \pm SEM $\mathrm{N}=20$.

\begin{tabular}{|l|l|c|c|c|c|l|l|l|r|r|}
\hline \multicolumn{2}{|c|}{} & \multicolumn{3}{|c|}{ Pre training } & \multicolumn{5}{c|}{ Post training } \\
\hline Cell type & Fluorochrome & PBS & PMA & SI & PBS & p-value & PMIA & p-value & SI & p-value \\
\hline Gran & DHR & $26 \pm 1$ & $5128 \pm 416$ & $199.0 \pm 16$ & $26 \pm 1$ & $0.687(\mathrm{NS})$ & $5434 \pm 478$ & $0.642(\mathrm{NS})$ & $214.0 \pm 19$ & $0.554(\mathrm{NS})$ \\
\hline Gran & DHE & $50 \pm 3$ & $2917 \pm 177$ & $62.0 \pm 5$ & $50 \pm 2$ & $0.783(\mathrm{NS})$ & $3148 \pm 188$ & $0.382(\mathrm{NS})$ & $65.0 \pm 5$ & $0.621(\mathrm{NS})$ \\
\hline Mo & DHR & $290 \pm 26$ & $524 \pm 32$ & $2.1 \pm 0.2$ & $282 \pm 22$ & $0.816(\mathrm{NS})$ & $461 \pm 34$ & $0.195(\mathrm{NS})$ & $1.8 \pm 0.1$ & $0.267(\mathrm{NS})$ \\
\hline Mo & DHE & $73 \pm 4$ & $829 \pm 48$ & $12.2 \pm 1.0$ & $71 \pm 3$ & $0.638(\mathrm{NS})$ & $752 \pm 38$ & $0.255(\mathrm{NS})$ & $11.2 \pm 0.8$ & $0.455(\mathrm{NS})$ \\
\hline
\end{tabular}

NS $=$ non significance. Gran $=$ granulocytes. Mo $=$ monocytes. DHE $=$ dellydroethidium. DHR $=$ dihydrorhodamine 123. 
Examining the post-training blood samples demonstrated that both PBS (control samples) and PMA-stimulated samples, as well as the calculated stimulation index (SI=PMA-stimulated/ control) were all non-significantly changed compared to the pretraining samples.
Figure 1 illustrates the granulocyte basal ROS levels the ROS increase induced by PMA and the stimulation index (SI) before and after a strength training session measured with the DHR and the DHE probes. Total antioxidant status and catalase before and after the training session.

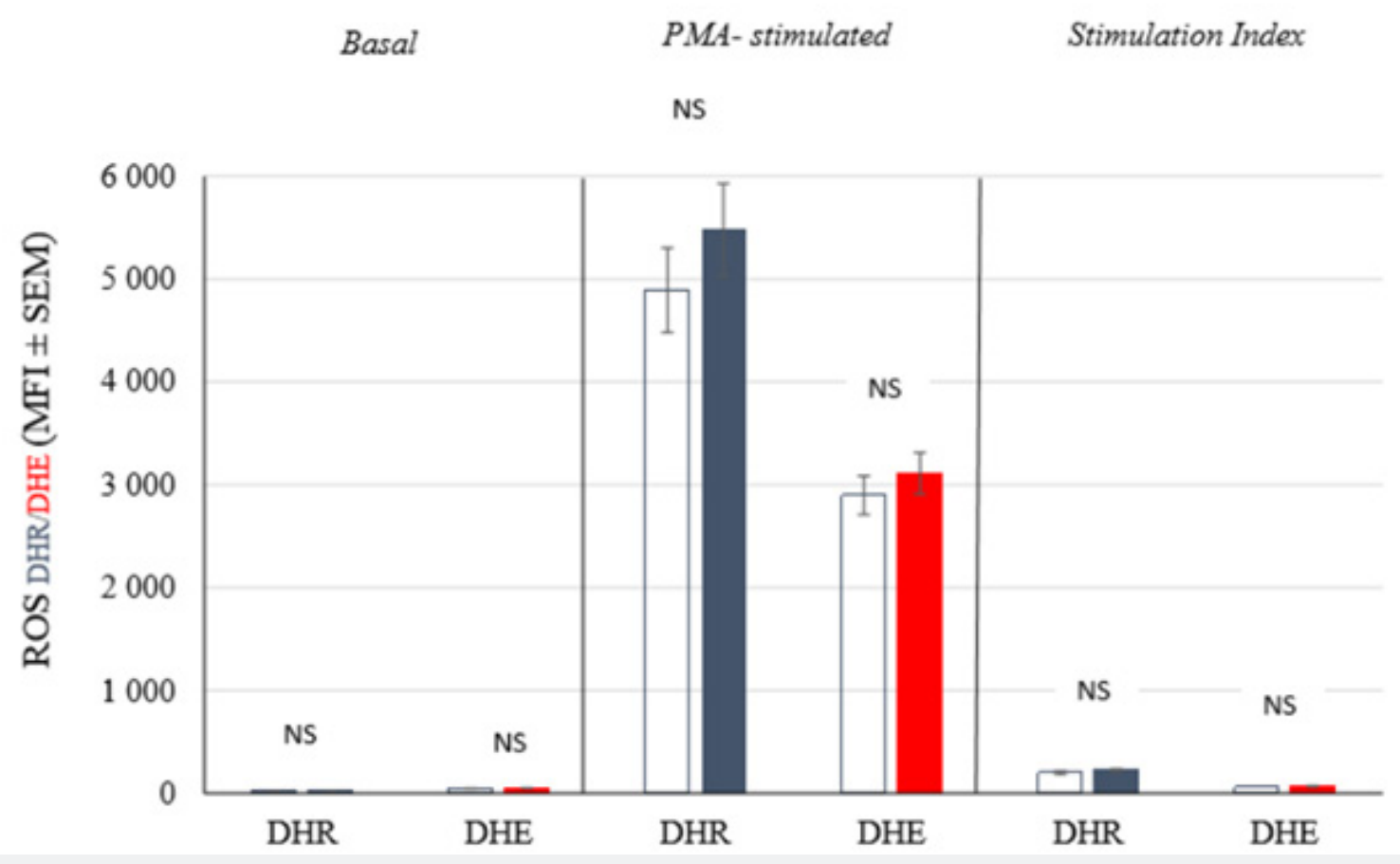

Figure 1: Basal and PMA - stimulated ROS-levels in granulocytes after a strength training session measured with the respective DHR- and $\mathrm{DHE}$ probe. Data are expressed as mean \pm Sem. $\mathrm{N}=20$. $\mathrm{NS}=$ non-significant. $\mathrm{DHR}=$ Blue colour. $\mathrm{DHE}=\mathrm{Red}$ colour. Open columns= pre - training. Filled columns $=$ post - training.

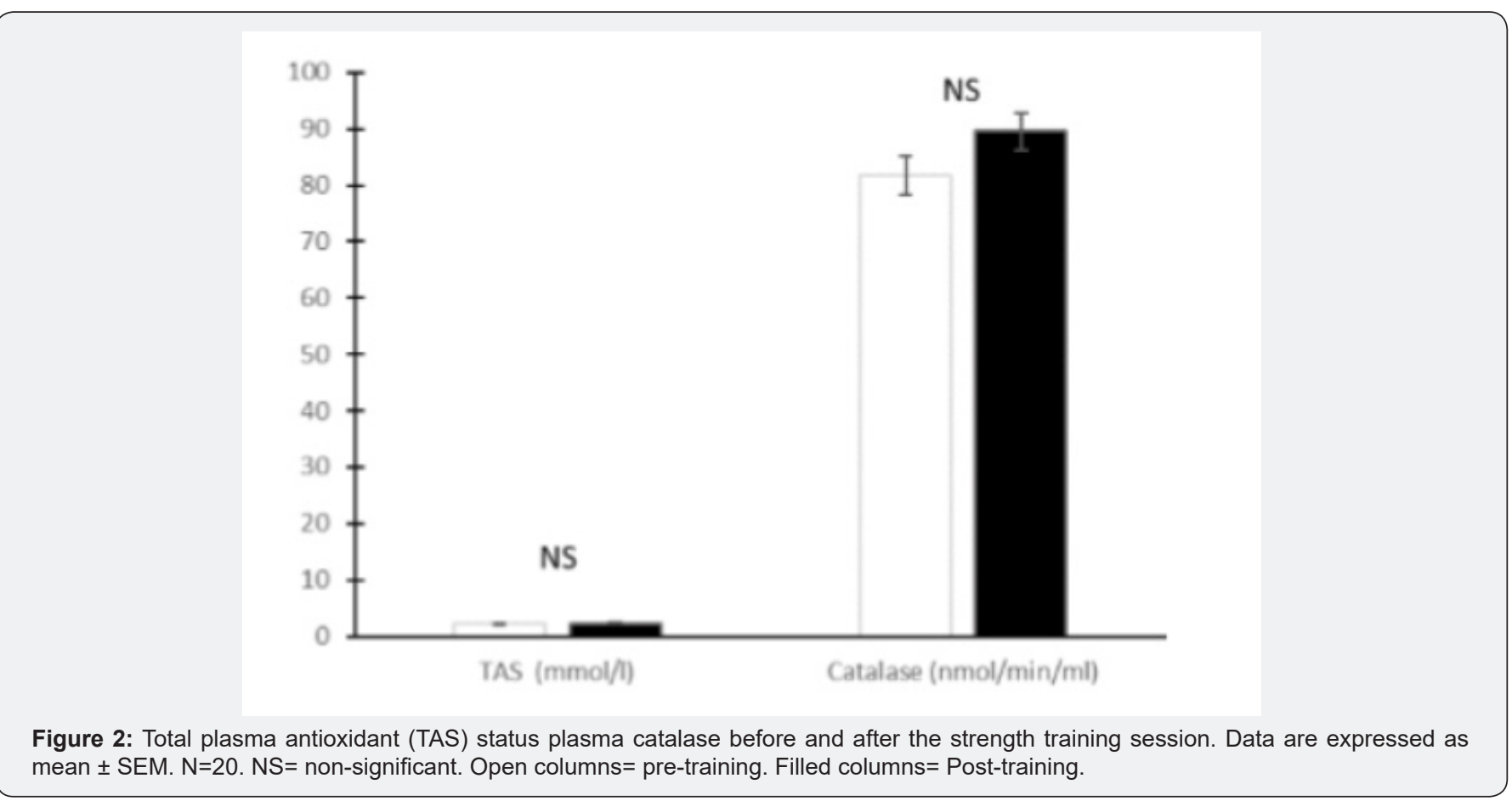


The total plasma antioxidant status and the levels of plasma catalase did no change after the training session (Figure 2).

\section{Discussion}

We have in the present study investigated the changes in selected haematological parameters, the leukocyte expression of ROS measured with flow cytometry using the DHE- and the DHR probes basal and after in vitro stimulation with PMA, total plasma antioxidant capacity and catalase in males and females' athletes before and after a strength training session.

We observed after the strength training session unchanged levels of total number of leukocytes. This is in contrast to what we observed in our study with the marathon and the half-marathon runners where we found a 3.2-fold and a 2.4- fold increase in leukocytes after the races [16]. Similarly, we observed a 1.6-fold increase of circulating leukocytes after a short term physical exercise until exhaustion (test of maximal oxygen consumption $\left(\mathrm{VO}_{2 \max }\right)$ ) [17]. Our former studies confirm earlier studies; changes in white blood cells are dependent of type of exercise and the intensity of the exercise $[1,2,5,7,18-20]$.

As in our earlier studies $[16,17,21]$ we have in this study used the highly sensitive flow cytometric method for measuring ROS generation in leukocytes. We observed after a $\mathrm{VO}_{2 \max }$ test a significant increase in ROS levels in leukocytes [17]. Based upon that observation, we infer that the leukocytes have been primed for ROS production during the primary phase. Upon prolonged running the ROS generation machinery in leukocytes seems to be temporal exhausted, more exhausted after a full marathon race than after the shorter half-marathon race [16]. In the present work on strength training, we could not observe significantly changes in the leukocyte ROS levels comparing pre-training values with post- training values. A marathonand a half marathon race is executed at an intensity where the production and utilisation of blood lactate ([La- $] \mathrm{b})$ is equal. The average running time in marathon race was $3 \mathrm{~h} 40 \mathrm{~min}$ and the half- marathon race was finished in $1 \mathrm{~h} 53 \mathrm{~min}$ for the women and $1 \mathrm{~h} 41 \mathrm{~min}$ for the men [16]. The test of $\mathrm{VO}_{2 \max } \mathrm{V}$ ends with at deflating of heart frequencies about 8-10 beats below the athletes' maximum heart frequency $\left(\mathrm{HF}_{\max }\right)$ and the test is over within $10 \mathrm{~min}$. The present strength training session consisted of nine exercises which were executed at $80 \%$ of One Repetition Maximum (1RM), 6-8 repetitions and 3 sets. The exercise session lasts for about 60 minutes in average. The reason for non-responses in leukocytes ROS is probably due to the short duration of the exercise session even though quite high heart frequencies were applicable after every single exercise. The total work load seems to be not high (intensity) or last long (duration) enough to influence the leukocyte generation of ROS.

The different levels of fitness form of the subjects in our studies could also be an explanation for the different responses of leukocyte generation of ROS. This in combination with the different types of exercise which were performed. The optimal situation would be to test the same persons executing all the different training regimes, but this is nearly impossible with active athletes/students who have their own training programme.

The total plasma antioxidant status level and catalase levels were not affected by the strength training session. We observed increased TAS levels after the marathon and the half-marathon race. The increase was higher in the marathon runners compared to the half- marathon runners, probably due to the duration of the activity [16]. The results corresponds also to what we observed in 10 cadets after a ranger-training course [21]. We observed no changes in TAS levels comparing values before and after the test of $\mathrm{VO}_{2 \max }$ [17]. A session of strength training was not enough (total intensity of the work) or last long enough (duration of the exercise) to influence any of the measured parameters. Based on this, a normal diet including daily recommended doses of vitamins and minerals seems to be suitable for performing this type of exercise.

\section{Conclusion}

One-hour strength training consisting of nine exercises performed at $80 \%$ of $1 \mathrm{RM} / 6-8$ repetitions/3 sets were not enough to influence the leukocyte production of ROS and the total plasma antioxidant system in trained students. Based on these findings, there is no rationale to advice athletes to ingest antioxidant supplementation in addition to their regular diet.

\section{Acknowledgement}

The authors would like to thank the participants who voluntarily participated in this project. A special thanks to MLT Lisbeth Saetre for excellent technical and practical assistance. Thanks to Oslo University Hospital for funding of the project and to Kristiania University College for economical support.

\section{References}

1. NP Walsh, M Gleeson, RJ Shepard (2011) Position statement. Part one: Immune function and exercise. Exercise Immunology Review 17: 6-63.

2. NP Walsh, M Gleeson, DB Pyne (2011) Position statement. Part two: Maintaining immune health. Exercise Immunology Review 17: 64-103.

3. PO Åstrand (2003) Textbook of Work Physiology. Physiological Bases of Exercise, HADK Rodahl, SB Strømme, (Eds.); Human Kinetics.

4. PO Åstrand, Rodahl K (1986) Textbook of Work Physiology, (3 $3^{\text {rd }}$ Edn); McGraw-Hill Book Company, Singapore.

5. NP Walsh (2018) Recommendations to maintain immune health in athletes. European Journal of Sport Sciences 11: 1-12.

6. JA Woods, JM Davies, JA Smith (1999) Exercise and cellular innate immune function. Medicine \& Science in Sports \& Exercise 31(1): 57 66.

7. DC Nieman, S Nehlsen-Caranella (1992) Effects of Endurance Exercise on the Immune Response, in Endurance in Sport, RJ Shepard, PO Åstrand, (Eds.); Blackwell Science Ltd, London, p. 487-504.

8. LM Wentz, MD Ward, C Potter (2018) Increased Risk of Upper Respiratory Infection in Military Recruits Who Report Sleeping Less Than $6 \mathrm{~h}$ per night. Military Medicine. 
9. SL Williams, NA Strobel, LA Lexis (2006) Antioxidant requirements of endurance athletes: Implications for health. Nutrition Reviews 64(3): 93-108.

10. Thirupathi A, RA Pinho (2018) Effects of reactive oxygen species and interplay of antioxidants during physical exercise in skeletal muscles. Journal of Physiology and Biochemistry.

11. E Fehrenbach, H Northoff (2001) Free radicals, exercise, apoptosis, and heat shock proteins. Exercise Immunology Review 7: 66-89.

12. Pingitore G, P Lima, F Mastorci (2015) Exercise and oxidative stress: potential effects of antioxidant dietary strategies in sports. Nutrition 31(7-8): 916-922.

13. RJ Maughan, LM Bruke, J Dvorak (2018) IOC Consensus Statement: Dietary Supplements and the High-Performance Athlete. International journal of sport nutrition and exercise metabolism 28(2): 104-125.

14.S Bermon, LM Castell, PC Calder (2017) Consensus Statement Immunonutrition and Exercise. Exercise Immunology Review 23: 8-50.

15. Stritesky Larssen K, Lyberg T (2006) Oxidative status--age- and circadian variations? --a study in leukocytes/plasma. Neuro Endocrinol Lett 27(4): 445-452.
16.HG Nielsen, IA Hagberg, T Lyberg (2004) Marathon running leads to partial exhaustion of ROS-generating capacity in leukocytes. Medicine and Science in Sports and Exercise 36(1): 68-73.

17.HG Nielsen, OH Skjonsberg, T Lyberg (2008) Effect of antioxidant supplementation on leucocyte expression of reactive oxygen species in athletes. Scandinavian journal of clinical and laboratory investigation 68(7): 526-533

18.DC Nieman (1997) Immune response to heavy exertion. Journal of Applied Physiology 82(5): 1385-1944.

19. MK Fagerhol, HG Nielsen, A Vetlesen (2005) Increase in plasma calprotectin during long-distance running. Scandinavian Journal of Clinical \& Laboratory Investigation 65(3): 211-220.

20.NP Walsh, SJ Oliver (2016) Exercise, immune function and respiratory infection: An update on the influence of training and environmental stress. Immunology Cell Biololgy 94(2): 132-139.

21.HG Nielsen, PK Opstad, T Lyberg (2007) LeuCAM and reactive oxygen species during long-term exercise combined with sleep and energy deficiency. Medicine and Science in Sports Exercise 39(2): 275-282. 